\title{
Respiratory syncytial virus and adenovirus in acute lower respiratory infections in hospitalized infants and children
}

\author{
Salvatore Barberi ${ }^{1}$, Mario Barreto ${ }^{1}$, Francesco La Penna ${ }^{1}$, Bernardina Mazzarella ${ }^{1}$, \\ Maria-Elena Liverani ${ }^{1}$, Ottavia De Luca ${ }^{2}$, Maurizio Simmaco ${ }^{2}$, Maria Pia Villa ${ }^{1}$ \\ ${ }^{1}$ NESMOS Department, Faculty of Medicine and Psychology, Pediatric Unit, Sant'Andrea Hospital, University “La Sapienza”, Rome, \\ Italy \\ ${ }^{2}$ NESMOS Department, Faculty of Medicine and Psychology, DIMA Unit (Advanced Molecular Diagnostics), Sant'Andrea Hospital, \\ University "La Sapienza", Rome, Italy \\ Email: salvatorebarberi@hotmail.com
}

Received 11 October 2011; revised 22 November 2011; accepted 21 December 2011

\begin{abstract}
Background: Lower respiratory tract infection (LRTI) is a main cause of hospitalization in infants and children. These patients' nasopharyngeal swabs more frequently contain respiratory syncytial virus (RSV) than human adenovirus (HAdV) in cold seasons; recent data suggest that oropharyngeal swabs more frequently contain HAdV than RSV. Knowing more about virus detection with oropharyngeal swabs, seasonal fluctuations and age-related distribution of RSV and HAdV would help treat children hospitalized for LRTI more effectively. We investigated the seasonal virus-related frequency (as assessed by oropharyngeal swabs) and environmental and clinical features in infants and children hospitalized for LRTI from autumn to spring. Methods: We studied 98 subjects hospitalized for LRTI in our Pediatric Unit, from November 2006 to May 2007. RSV and HAdV in oropharyngeal swabs were assessed by real-time polymerase chain reaction (PCR) assay. Results: PCR assays more frequently detected HAdV $(29.6 \%$ of patients) than RSV (25.5\%). The seasonal incidence also differed (RSV, narrow peak in December and HAdV, wide peak from April to May). Most patients infected with RSV were aged 2 yrs or younger (23/25: $88 \%$ ); no difference was found in age between subjects who tested HAdV-positive (13/29: 45\%) or undetectable-virus (23/44: 52\%). Bronchiolitis was more frequently associated with RSV than HAdV or undetectable-virus oropharyngeal swabs. No difference was found in risk factors (school attendance, atopic parents, exposure to cigarette smoking, home dampness or exposure to animals) or clinical features (vital parameters or duration of hospital stay) among the three viral groups. Conclusions: Our findings show a high frequency of HAdV-positive oropharyngeal swabs during acute LRTIs in infants and children and dif-
\end{abstract}

ferences in the seasonal distribution of RSV and HAdV in Rome.

Keywords: RSV; Adenovirus; Bronchiolitis; Children; Pneumonia

\section{INTRODUCTION}

Respiratory syncytial virus (RSV) is a major cause of lower respiratory tract infection (LRTI) and hospitalization especially in infants but also in children [1,2]. The most common clinical presentation of RSV infection in infants is bronchiolitis [3-5].

Several studies on clinical symptoms, incidence and seasonal variations in RSV infections show yearly epidemics, whose onset varies with latitude, altitude and climate. In Mediterranean areas RSV outbreaks happen mainly in winter whereas in tropical countries they happen in the rainy months [6-9]. RSV epidemics typically alternate between small, late-season outbreaks and large, early-season outbreaks [9-18].

LRTIs in infants and children also arise from human adenovirus (HAdV) [19]. There are at least 51 immunologically distinct human HAdV serotypes (6 species: HAdV A through F) associated with infections ranging from respiratory disease (HAdV-B1, C, E), urinary tract disease (HAdV-B2), and keratoconjunctivitis (HAdV- D), to gastroenteritis (HAdV-F serotypes 40 and 41) [20]. The few available data on HAdV epidemics suggest a non-seasonal (perennial) distribution [21,22]. More information on infants and children hospitalized for HAdVrelated LRTI would help improve therapy and might also reduce health resources in a pediatric ward.

Sampling could influence the frequency of detection of RSV and HAdV [23]. Recently it has been reported that oropharyngeal swabs were more sensitive than nasopharyngeal swabs for detecting HAdV whereas com- 
parable for detecting RSV [23].

Our primary aim in this prospective single-center study conducted in an Italian academic children's hospital was to investigate the seasonal virus-related frequency (as assessed by oropharyngeal swabs) and environmental and clinical features in infants and children hospitalized for LRTI from autumn to spring in the Rome area. We hypothesized a similar seasonal distribution of RSV and HAdV-related LRTIs with a higher frequency of RSV-related LRTI. Because bronchiolitis is an agerelated LRTI, we analyzed data separately for patients 2 yr old or younger and patients older than 2 yr. Clinical findings included wheezing, subcostal recession, respiratory rate, and pulse-oximetry values on admission; treatment included oxygen therapy, oral corticosteroid therapy during hospitalization.

\section{MATERIALS AND METHODS}

\subsection{Subjects}

Infants and children hospitalized in the Pediatric Unit of S. Andrea Hospital, Rome, from November 2006 to May 2007 for LRTI (bronchiolitis, pneumonia or bronchitis) were consecutively and prospectively recruited for the study. Bronchiolitis was defined in the presence of tachypnea, wheezing, cough and crackles preceeded by rhinitis and absence of documented alveolar consolidation by both chest radiographs and clinical examination; pneumonia was defined as alveolar consolidation (chest radiographs, auscultation of rales and/or bronchial breathing); bronchitis was defined in a child with cough and at least one of the following symptoms: fever, wheezing, or dyspnea without radiological and clinical suspicion of bronchiolitis or pneumonia [24-26]. Exclusion criteria were congenital cardiac disease and severe airway anomaly. Parents gave signed consent for their children to participate and the institutional review board approved the procedures.

\subsection{Questionnaires and Clinical Records}

At study entry, parents were asked to complete a questionnaire on patient history (gestational age and weight, Apgar index, breast feeding, previous respiratory and gastrointestinal diseases, age, weight and height at admission to hospital); family history (atopy, respiratory and gastrointestinal diseases, number of children in the family, preschool age siblings); and environmental pattern (cigarette smoke, dampness, and domestic animals). Two physicians, unaware of viral (oropharyngeal swab) results completed clinical records and physical examinations in the pediatric ward. Clinical findings were collected at admission on wheezing, subcostal recession, respiratory rate, and pulse-oximetry values $\left(\mathrm{SaO}_{2}\right)$. Treat- ment during hospitalization was assessed from data on oxygen therapy, oral corticosteroid therapy and duration of hospitalization.

\subsection{Laboratory Tests}

Oropharyngeal swabs were obtained from all participants on entry to the study for RSV and HAdV nucleic acid assay. Samples from oropharyngeal swabs were diluted in $200 \mu \mathrm{L}$ of phosphate buffered saline (PBS) $1 \mathrm{X}$ and nucleic acids were extracted with the High Pure Viral Nucleic Acid Kit (Roche) according to the manufacturer's instructions. This method extracts DNA and RNA simultaneously. All samples were assayed with real-time polymerase-chain reaction (PCR).

RSV was quantified with the SuperScriptTM III Platinum ${ }^{\circledR}$ One-Step Quantitative real-time-PCR System (Invitrogen) and an oligo-mix (Arrow Diagnostics) containing two specific probes, one for RSV-A and the other for RSV-B [1-7,14], both labeled with fluorescent (FAM) and quencher dyes. Thermal cycling comprised an initial 15 minute incubation at $50^{\circ} \mathrm{C}$ (to synthesize cDNA from viral RNA) followed by denaturation at $95^{\circ} \mathrm{C}$ for 2 minutes and 35 cycles at $95^{\circ} \mathrm{C}$ for 15 seconds and at $58^{\circ} \mathrm{C}$ for 30 seconds. During this last step fluorescence was detected in the FAM channel.

For HAdV we used Premix Ex TaqTM [(Perfect Real Time) (Taqara Bio Inc.)] and an oligo-mix (Arrow Diagnostics) containing specific probes for the main adenovirus serotypes $[27,28]$ labeled with fluorescent (FAM) and quencher dyes. The PCR cycling program consisted of an initial denaturation step at $95^{\circ} \mathrm{C}$ for 30 seconds and 35 cycles at $95^{\circ} \mathrm{C}$ for 15 seconds, at $58^{\circ} \mathrm{C}$ (FAM-channel data acquistion) for 30 seconds and $72^{\circ} \mathrm{C}$ for 10 seconds.

DNA fragments were amplified, RNA and DNA detected and data analyzed with the Rotor-Gene 3000TM Real Time PCR System (Corbett Research) at the Diagnostic Molecular Unit of S. Andrea Hospital.

\subsection{Statistical Analysis}

Data were analyzed overall and separately for patients 2 yr old or younger and patients older than 2 yr. Normallydistributed variables were described as means and standard deviations; skewed variables were expressed as medians and interquartile ranges (IQR). Student $T$ test or the Mann-Whitney U test were used to compare two groups of data and an analysis of variance (ANOVA) with post hoc Scheffè or the Kruskal-Wallis test was used to compare more than two groups, when appropriate. Chi square test and Fischer's corrections were used to compare $2 \times 2$ and Bonferroni test to evaluate $2 \times \mathrm{n}$ contingency tables. $P$ values less than 0.05 were considered statistically significant. Data were analyzed with the "Statistica release 6" package (StatSoft Corp., Tulsa, OK, USA). 


\section{RESULTS}

A total of 98 consecutive children (girls/boys: 41/57) hospitalized in the Pediatric Unit of S. Andrea Hospital, Rome, from November 2006 to May 2007 were prospectively enrolled in the study. Mean age at study entry was 33.8 months (median 17 months, IQR 39.0). None of the patients were excluded.

Of the 98 oropharyngeal swabs examined 25 (25.5\%) were RSV positive and 29 (29.6\%) HAdV positive by real-time PCR; the remaining 44 swabs (44.9\%) were undetectable for viruses. RSV infections peaked in December (14/19: $74 \%$ of total monthly swabs), decreased in January (11/25: 44.0\%) and ended by February. HAdV positive findings in oropharyngeal swabs increased from December (1/19: $5.3 \%)$ to January $(2 / 25: 8.0 \%)$, rose sharply until it peaked in April (6/9: 66.7\% of total monthly swabs) and remained high in May (7/11: 63.6\%) (Figure 1).

Hospitalized patients with RSV positive swabs were younger than those in the other two viral groups: median age (IQR) 5.0 months (13.0) vs 25.0 (30.0) for HAdV and 21.0 (73.8) for undetectable-viruses. The age-related distribution of respiratory diseases therefore differed among the three viral groups (Table 1). In patients with a diagnosis of bronchiolitis, RSV positive swabs were more frequent than HAdV positive or undetectable-virus oropharyngeal swabs ( $\mathrm{p}=0.00011$ by Bonferroni Test). Conversely, in patients with pneumonia or bronchitis, no significant percentage differences were found between the three viral-related groups (Table 1).

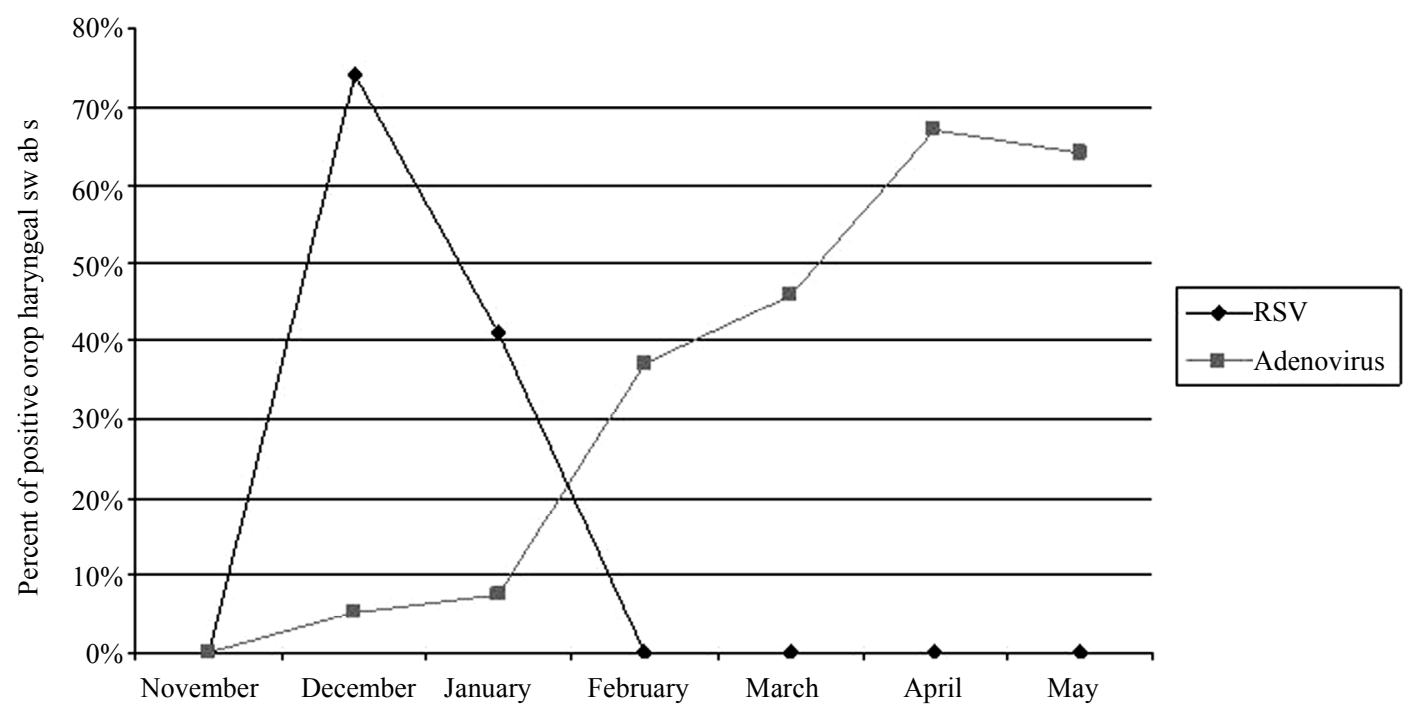

Figure 1. Monthly distribution of virus-positive oropharyngeal swabs (out of total oropharyngeal swabs) in the 98 infants and children prospectively enrolled in the study.

Table 1. Percentage distribution of positive oropharyngeal swabs in the 98 patients by age range and diagnosis.

\begin{tabular}{ccccc}
\hline \multirow{2}{*}{ LRTI } & AGE & $\begin{array}{c}\text { RSV } \\
\leq 2 \mathrm{yr} n=22 \\
>2 \mathrm{yr}: \mathrm{n}=3\end{array}$ & $\begin{array}{c}\text { HadV } \\
\leq 2 \mathrm{yr}: \mathrm{n}=13 \\
>2 \mathrm{yr}: \mathrm{n}=16\end{array}$ & $\begin{array}{c}\text { UNDETECTABLE } \\
\leq 2 \mathrm{yr}: \mathrm{n}=23 \\
>2 \mathrm{yr}: \mathrm{n}=21\end{array}$ \\
\hline \multirow{3}{*}{ Bronchiolitis } & $14(63.6 \%)^{*}$ & $3(23.1 \%)$ & $7(30.4 \%)$ \\
& $>2 \mathrm{yr}$ & $0(0.0 \%)$ & $0(0.0 \%)$ & $0(0.0 \%)$ \\
& total & $14(56.0 \%)$ & $3(10.3 \%)$ & $7(15.9 \%)$ \\
\multirow{3}{*}{ Pneumonia } & $52 \mathrm{yr}$ & $5(22.7 \%)$ & $2(15.4 \%)$ & $5(63.6 \%)$ \\
& $>2 \mathrm{yr}$ & $3(100.0 \%)$ & $5(31.2 \%)$ & $10(47.6 \%)$ \\
& total & $8(32.0 \%)$ & $7(24.1 \%)$ & $15(34.1 \%)$ \\
Bronchitis & $\leq 2 \mathrm{yr}$ & $3(13.6 \%)$ & $8(61.5 \%)$ & $11(63.6 \%)$ \\
& $>2 \mathrm{yr}$ & $0(0.0 \%)$ & $11(68.7 \%)$ & $11(52.4 \%)$ \\
& total & $3(12.0 \%)$ & $19(65.5 \%)$ & $22(50.0 \%)$ \\
\hline
\end{tabular}

LRTI: lower respiratory tract infection; RSV: respiratory syncytial virus; HAdV: adenovirus; UNDETECTABLE: negative swabs for RSV or HadV. ${ }^{*} \mathrm{p}<0.002$ vs HAdV and UNDETECTABLE groups by Bonferroni test. 
Risk factors for LRTIs including parental atopy and environmental characteristics (exposure to cigarette smoking, home dampness, pet ownership, and nursery or school attendance) were similar in the RSV, HAdV and undetectable-virus groups, as were patient characteristics (data not shown). No between-group differences were found for respiratory rate at hospital admission and duration of hospitalization in infants (2 yr old or younger) or children (older than $2 \mathrm{yr}$ ). Nor were between group differences found for pulse-oximetry values $\left(\mathrm{SaO}_{2}\right)$ in infants although in children, $\mathrm{SaO}_{2}$ values were lower in subjects who had RSV infection than in those who had $\mathrm{HAdV}$ infection or undetectable-virus infection $(92.7 \pm$ $3.8 \%$ vs $96.9 \pm 1.0 \%$ vs $96.4 \pm 1.6 \%)(p=0.002)$.

Also in children, oxygen therapy and corticosteroid therapy was needed less often for children with HAdV infections than those with RSV infections or undetectable-virus infections (Figures 2(a)-(b)).

\section{DISCUSSION}

In this prospective single-center study the seasonal virus-related frequency differed markedly in infants and children hospitalized for LRTI (bronchiolitis, pneumonia and bronchitis) during the winter-spring season 2006 to 2007. Whereas RSV-related LRTIs peaked sharply in winter (December) HAdV-related infections had a wider peak in spring (April and May). The frequency of HAdVpositive oropharyngeal swabs was high in our study sample, about $30 \%$. To our knowledge this is the highest

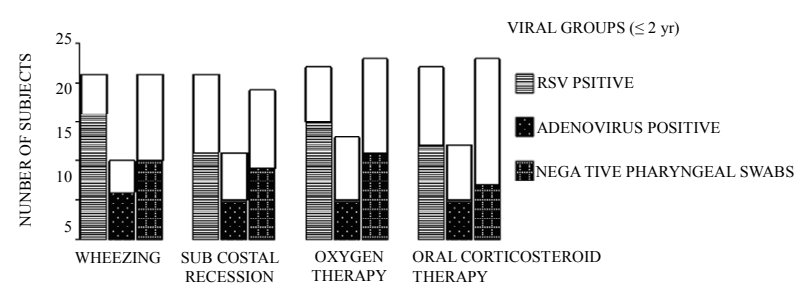

(a)

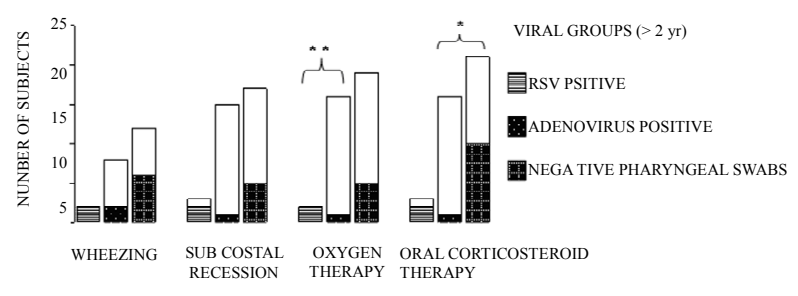

(b)

Figure 2. Clinical findings and therapy by viral oropharyngeal swabs. A: subjects aged 2 yr or less; B: subjects older than 2 yr. Unfilled columns represent asymptomatic subjects. ${ }^{*} \mathrm{p}<$ 0.05 adenovirus positive vs undetected-virus swabs. ${ }^{* *} \mathrm{p}<0.01$ respiratory syncytial virus-positive vs adenovirus-positive swabs. Contingency tables were used with post hoc Bonferroni's correction. reported prevalence of HAdV-related LRTI in a similar hospitalized population. RSV accounted for bronchiolitis in about two-thirds of patients aged two years or younger and for pneumonia in about one-third of those older that 2 years. Environmental characteristics were evenly distributed in our patients whereas clinical features depended mainly on age.

The peak RSV incidence we found in winter agrees with previous research showing that RSV incidence peaks are common in the cold months and vary with geographical position [29]. Peaks have been reported in December [30] January [31], February [8,30,32], and March [33]. Below the Ecuatorial line, RSV peaks have been described in May [34] and July [22]. A previous Italian study described an epidemic peak of RSV in February with a prevalence of $52 \%$ in hospitalized children with LRTI [32]. In another study conducted in Korea in hospitalized children $5 \mathrm{yr}$ or younger, the number of RSV infections increased during late fall and peaked between November and January [35].

The $29.6 \%$ rate of HAdV in our children's oropharyngeal swabs is about twice the reported seasonal incidence for this virus using nasopharyngeal swabs, which varies from $2 \%$ to $17.9 \%$ [31,34-38]. Whether our high percent HAdV rate arises from methodological or geographical differences remains to be elucidated. Monthly peaks of HAdV incidence are reported in April [31], April and September [34] but at the end of winter and beginning of spring in the southern hemisphere [22]. In the study by Hong et al, HAdV serotype 7 (associated with the highest morbidity) peaked sharply in May 1996 [36]. Two studies in Seoul, Korea, from 1990 to 1998 [36] and from 2000 to 2005 [35] reported that the monthly occurence of HAdV infections varied from one year to another [35, 36]. Data from future assessments, possibly addressing HAdV serotypes, will enable us to estimate how the annual incidence of HAdV fluctuates in our geographical setting.

When we investigated the various subject or environmental characteristics known to favor the onset of viral LRTIs (male sex, breast feeding, prematurity, atopy, exposure to tobacco smoke, crowding, dampness and pets at home) [29,39-41], we found that none of them differed in patients infected with RSV, HAdV or any of these two infections. Further studies enrolling a larger study sample are needed to assess risk factors for specific viral LRTIs between age-matched patients.

Our finding that most patients infected with RSV were infants (22/25: 88\%) and most of them (19/22: 86\%) had either bronchiolitis or pneumonia fits in well with current knowledge that RSV predominantly infects subjects aged $2 \mathrm{yr}$ or younger [8,31,33,34,37,38]. HAdV infections appear to be less age-dependent $[35,36,38]$. Accordingly, of the $29 \mathrm{HAdV}$-positive subjects, 13 (45\%) were infants, 
of whom $5(38.5 \%)$ had either bronchiolitis or pneumonia. In children, HAdV was more frequently related to bronchitis. Unlike others [34,36], we did not find that subjects infected with HAdV were more prone to pneumonia than those infected with RSV. The discordant results probably depend on differences in environmental, host (e.g. genetic) and viral factors (e.g. serotypes).

A finding of interest to clinical pediatric practice concerns our patients' clinical conditions on admission to hospital. Our children (older than 2 yr) with HAdV needed oxygen therapy and oral corticosteroids less often than their RSV-positive and negative counterparts, whereas among our infants, assessment of clinical conditions yielded no significant between-group differences. Our findings agree with a similar study describing no differences in clinical features among the same viral groups we report [22]. Conversely, some reports estimate that as many as $50 \%$ of patients infected with HAdV have to be admitted to pediatric intensive care units $[42,43]$. Whether the severity of virus-related LTRIs we observed in a single observation period holds true in a longer observation needs confirming in further assessments.

Sensitivity for virus detection in our study could have been improved by including both oropharyngeal and nasopharyngeal swabs. However, using both swabs adds costs and patient discomfort. Results from a recent study suggest that a single-swab, whether oropharyngeal or nasopharyngeal could be acceptable for respiratory disease surveillance [23]. For the preceeding reasons and the relative scarcity of our population sample, our results should be interpreted with caution.

In conclusion, we isolated an unexpectedly high proportion of HAdV in oropharyngeal swabs from infants and children hospitalized in our pediatric unit during spring 2007. RSV infections peaked in December 2006. Both HAdV and RSV infections accounted for a high morbidity, particularly bronchiolitis and pneumonia. If we are to manage LRTI in hospitalized children more efficiently, we need more information on the various types of viral infections responsible for hospital admission and their severity.

\section{REFERENCES}

[1] Filippel, M.B. and Rearick, T. (1993) Respiratory syncytial virus. Nursing Clinics of North America, 28, 651671.

[2] Sigurs, N., Bjarnason, R. and Sigurbergsson, F. (2000) Respiratory syncytial virus bronchiolitis in infancy is an important risk factor for asthma and allergy at age 7 . American Journal of Respiratory and Critical Care Medicine, 161, 1501-1507.

[3] Simoes, EA. (1999) Respiratory syncytial virus infection. Lancet, 354, 847-852.
[4] Hall, C.B. (2001) Respiratory syncytial virus and parainfluenza virus. New England Journal of Medicine, 344, 1917-1928. doi:10.1056/NEJM200106213442507

[5] Leader, S. and Kohlhase, K. (2003) Recent trends in severe respiratory syncytial virus (RSV) among US infants 1997 to 2000. Journal of Pediatrics, 143, S127-S132. doi:10.1067/S0022-3476(03)00510-9

[6] Centers for Disease Control and Prevention (CDC) (2004) Respiratory syncytial virus activity-United States 20032004. Morbidity and Mortality Weekly Report, 53, 11591160.

[7] Robertson, S.E., Roca, A., Alonso, P., et al. (2004) Respiratory syncytial virus infection: Denominator-based studies in Indonesia, Mozambique, Nigeria and South Africa. Bulletin World Health Organization, 82, 914-922.

[8] Medici, M.C., Arcangeletti, M.C., Merolla, R., et al. and The "Osservatorio VRS" Study Group (2004) Incidence of respiratory syncytial virus infection in infants and young children referred to the emergency departments for lower respiratory tract diseases in Italy. Acta Bio-Medica, 75, 26-33.

[9] Stensballe, L.G., Devasundaram, J.K. and Simoes, E.A. (2003) Respiratory syncytial virus epidemics: The ups and downs of a seasonal virus. Pediatrics Infectious Disease Journal, 22, S21-S32. doi:10.1097/01.inf.0000053882.70365.c9

[10] Van Der Sande, M.A., Goetghebuer, T., Sanneh, M., et al. (2004) Seasonal variation in respiratory syncytial virus epidemics in the Gambia, West Africa. Pediatrics Infectious Disease Journal, 23, 73-74. doi:10.1097/01.inf.0000105183.12781.06

[11] Mullins, J.A., Lamonte, A.C., Bresee, J.S., et al. (2003) Substantial variability in community respiratory syncytial virus season timing. Pediatrics Infectious Disease Journal, 22, 857-862.

doi:10.1097/01.inf.0000090921.21313.d3

[12] Duppenthaler, A., Gorgievski-Hrisoho, M., Frey, U., et al. (2003) Two-year periodicity of respiratory syncytial virus epidemics in Switzerland. Infection, 31, 75-80. doi:10.1007/s15010-002-3124-8

[13] Weigl, J.A., Puppe, W. and Schmitt, H.J. (2002) Seasonality of respiratory syncytial virus-positive hospitalizations in children in Kiel, Germany, over a 7-year period. Infection, 30, 186-192. doi:10.1007/s15010-002-2159-1

[14] Eriksson, M., Bennet, R., Rotzen-Ostlund, M., et al. (2002) Population-based rates of severe respiratory syncytial virus infection in children with and without risk factors, and out-come in a tertiary care setting. Acta Paediatrica, 91, 593-598. doi:10.1111/j.1651-2227.2002.tb03282.x

[15] Lyon, J.L., Stoddard, G., Ferguson, D., et al. (1996) An every other year cyclic epidemic of infants hospitalized with respiratory syncytial virus. Pediatrics, 97, 152-153.

[16] Chang, P.W.K., Chew, F.T., Tan, T.N., et al. (2002) Seasonal variation in respiratory syncytial virus chest infections in the tropics. Pediatric Pulmonology, 34, 47-51. doi:10.1002/ppul.10095

[17] Waris, M. (1991) Pattern of respiratory syncytial virus epidemics in Finland: two-year cycles with alternating 
prevalence of groups A and B. Journal of Infectious Diseases, 163, 464-469. doi:10.1093/infdis/163.3.464

[18] Medici, M.C., Arcangeletti, M.C., Rossi, G.A., et al. and The "Osservatorio VRS" Study Group (2006) Four year incidence of respiratory syncytial virus infection in infants and young children referred to emergency departments for lower respiratory tratc disease in Italy: The "Osservatorio VRS" Study (2000-2004). The New Microbiologica, 29, 35-43.

[19] Moro, M.R., Bonville, C.A., Suryadevara, M., et al. (2009) Clinical Features, adenovirus types, and local production of inflammatory mediators in adenovirus infections. Pediatrics Infectious Disease Journal, 28, 376-380. doi:10.1097/INF.0b013e31819075a6

[20] Zhang, Y. and Bergelson, J.M. (2005) Adenovirus receptors. Journal of Virology, 79, 12125-12131. doi:10.1128/JVI.79.19.12125-12131.2005

[21] Weissenbacher, M., Carballal, G., Avila, M., et al. (1990) Etiologic and clinical evaluation of acute lower respiratory tract infections in young Argentinian children: An overview. Reviews of Infectious Diseases, 12, S889-S898. doi:10.1093/clinids/12.Supplement 8.S889

[22] Videla, C., Carballai, G., Misirlian, A., et al. (1998) Acute lower respiratory infections due to syncytial virus and adenovirus among hospitalized children from Argentina. Clinical and Diagnostic Virology, 10, 17-23. doi:10.1016/S0928-0197(98)00017-8

[23] Kim, C., Ahmed, J.A., Eidex, R.B., et al. (2011) Comparison of nasopharyngeal and oropharyngeal swabs for the diagnosis of eight respiratory viruses by real-time reverse transcription-pcr assays. PLOS ONE, 6, 1-6.

[24] American Academy of Pediatrics Subcommittee on Diagnosis and Management of Bronchiolitis (2006) Diagnosis and management of bronchiolitis. Pediatrics, 118, 1774-1793. doi:10.1542/peds.2006-2223

[25] Madhi, S.A., Ludewick, H., Kuwanda, L., et al. (2007) Seasonality, incidence, and repeat human metapneumovirus lower respiratory tract infections in an area with a high prevalence of human immunodeficiency virus type-1 infection. Pediatrics Infectious Disease Journal, 26, 693-699. doi:10.1097/INF.0b013e3180621192

[26] Klig, J.E. (2006) Office pediatrics: Current perspectives on the outpatient evaluation and management of lower respiratory infections in children. Current Opinion in $\mathrm{Pe}$ diatrics, 18, 71-76. doi:10.1097/01.mpo.0000192520.48411.fa

[27] Hierholzer, J.C. (1995) Adenoviruses. In: Lennette, E.H., Lennette, D.A. and Lennette, E.T., Eds., Diagnostic Procedures for Viral, Rickettsial, and Chlamydial Infections, American Public Health Association, Washington, 169188.

[28] Raty, R., Kleemola, M., Melen, K., et al. (1999) Efficacy of PCR and other diagnostic methods for the detection of respiratory adenoviral infections. Journal of Medical Virology, 59, 66-72.

doi:10.1002/(SICI)1096-9071(199909)59:1<66::AID-JM V11>3.0.CO;2-\#

[29] Simoes, E.A. and Carbonell-Estrany, X. (2003) Impact of severe disease caused by respiratory syncytial virus in children living in developed countries. Pediatrics Infectious Disease Journal, 22, S13-S18. doi:10.1097/01.inf.0000053881.47279.d9

[30] Midulla, F., Scagnolari, C., Bonci, E., et al. (2010) Respiratory syncytial virus, human bocavirus and rhinovirus bronchiolitis in infants. Archives of Disease in Childhood, 95, 35-41. doi:10.1136/adc.2008.153361

[31] Fabbiani, M., Terrosi, C., Martorelli, B., et al. (2009) Epidemiological and clinical study of viral respiratory tract infection in children from Italy. Journal of Medical Virology, 81, 750-756. doi:10.1002/jmv.21457

[32] Lanari, M., Giovannini, M., Giuffré, L., et al. and the Investigators R.A.DA.R. Study Group (2002) Prevalence of respiratory syncytial virus infection in Italian infants hospitalized for acute lower respiratory tract infections, and association between respiratory syncytial virus infection risk factors and disease severity. Pediatric Pulmonology, 33, 458-465. doi:10.1002/ppul.10047

[33] Rossi, G.A., Medici, M.C., Merolla, R. and The "Osservatorio VRS" Study Group (2005) Incidence of respiratory syncytial positivity in young Italian children referred to the emergency departements for lower respiratory tract infection over two consecutive epidemic seasons. Infection, 33, 18-24. doi:10.1007/s15010-005-4010-y

[34] Thomazelli, L.M., Vieira, S., Leal, A.L., et al. (2007) Surveillance of eight respiratory viruses in clinical samples of pediatric patients in southeast Brazil. Jornal de Pediatria, 83, 422-428. doi:10.1590/S0021-75572007000600005

[35] Choi, E.H., Lee, H.J., Kim, S.J., et al. (2006) The association of newly identified respiratory viruses with lower respiratory tract infection in Korean children, 2000-2005. Clinical Infection Disease, 43, 585-592. doi: $10.1086 / 506350$

[36] Hong, J.Y., Lee, H.J., Piedra, P.A., et al. (2001) Lower respiratory tract infections due to adenovirus in hospitalized Korean children: Epidemiology, clinical features and prognosis. Clinical Infection Disease, 32, 1423-1429. doi:10.1086/320146

[37] Mlinaric-Galinovic, G., Vilibic-Cavlek, T., Ljubin-Sternak, S., et al. (2009) Eleven consecutive years of respiratory syncytial virus outbreaks in Croatia. Pediatrics International, 51, 237-240. doi:10.1111/j.1442-200X.2008.02723.X

[38] Chan, P.W.K., Goh, A.Y.T., Chua, K.B., et al. (1999) Viral aetiology of lower respiratory tract infection young Malaysian children. Journal of Paediatrics and Child Health, 35, 287-290. doi:10.1046/j.1440-1754.1999.00359.x

[39] Simoes, E.A. (2008) RSV disease in the pediatric population: Epidemiology, seasonal variability, and long-term outcomes. Managed Care, 17, 18-19.

[40] Okiro, E.A., Ngama, M., Bett, A., et al. (2008) Factors associated with increased risk of progression to respiratory syncytial virus-associated pneumonia in young Kenyan children. Tropical Medicine \& International Health, 13, 914-926. doi:10.1111/j.1365-3156.2008.02092.x 
[41] Corsello, G., Di Carlo, P., Salsa, L., et al. (2008) Respiratory syncytial virus infection in a Sicilian pediatric population: Risk factors, epidemiology, and severity. Allergy and Asthma Proceedings, 29, 205-210. doi:10.2500/aap.2008.29.3101

[42] Morrow, B.M., Hatherill, M., Smuts, H.E., et al. (2006) Clinical course of hospitalised children infected with hu- man metapneumovirus and respiratory syncytial virus. Journal of Paediatrics and Child Health, 42, 174-178. doi:10.1111/j.1440-1754.2006.00825.x

[43] James, L., Vernon, M.O., Jones, R.C., et al. (2007) Outbreak of human adenovirus type 3 infection in a pediatric long-term care facility-Illinois, 2005. Clinical Infectious Diseases, 45, 416-420. doi:10.1086/519938 